\section{Abluftoxidation in Monolith-Katalysatoren mit periodischem Wechsel der Strömungsrichtung}

\section{Gerhart Eigenberger und Ulrich Nieken*}

\section{Herrn Professor Dr. Hanns Hofmann zum 65. Geburtstag}

Mit steigenden gesetzlichen Anforderungen zur Reduzierung des Schadstoffgehalts gewinnen Verfahren zur oxidativen Umwandlung von Schadstoffen in Abluft zunehmend an Bedeutung. Typische Aufgaben der Abluftreinigung sind gekennzeichnet durch hohe Luftdurchsätze und niedrige Schadstoffkonzentrationen; die adiabate Temperaturerhöhung der Totaloxidation beträgt häufig weniger als $100^{\circ} \mathrm{C}$. Das bedeutet, daß der Druckverlust in der Reinigungsanlage niedrig und der Wärmerücktausch zwischen $\mathrm{Zu}$ - und Ablauf hoch sein muß, um die Betriebskosten niedrig zu halten.

Im folgenden wird eine neuartige Betriebsweise der katalytischen Oxidation untersucht, die sich durch effektiven Wärmerücktausch, niedrigen Druckverlust und geringes Bauvolumen auszeichnet. Sie beruht auf der Benutzung von Monolith-Katalysatoren in Verbindung mit der von Matros und Mitarb. entwickelten Prozeßführung mit periodischem Wechsel der Strömungsrichtung [1,2]. Dabei wirkt jeweils der vordere und der hintere Teil des Katalysatorbettes als Regenerator-Wärmeaustauscher [3].

\section{Der eingeschwungene Zustand}

Die folgenden Beispiele beziehen sich auf Anwendungsfälle, bei denen die adiabate Temperaturerhöhung im Bereich von $50{ }^{\circ} \mathrm{C}$ liegt. Dazu muß im stationären Betrieb die Zulauftemperatur der Abluft auf einen Wert oberhalb der Anspringtemperatur der Reaktion eingestellt werden. Wird die Zulauftemperatur auf Umgebungstemperatur abgesenkt, so entwickelt sich eine sog. wandernde Brennzone [4-6]. Sie wandert langsam aus dem Reaktor, wobei zunächst noch Vollumsatz erhalten bleibt. Bei Betrieb mit wechselnder Strömungsrichtung
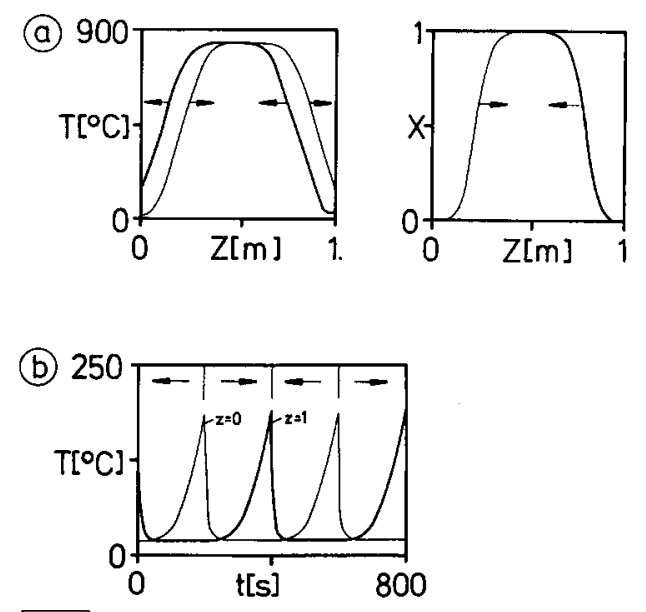

Sy 1009.1

Abb. 1. Eingeschwungener Zustand; a) Temperatur- und Umsatzprofil kurz vor Umschaltung der Strömungsrichtung, b) zeitlicher Verlauf der Austrittstemperatur.

\footnotetext{
* Prof. Dr. Ing. G. Eigenberger und Dipl.-Ing. U. Nieken, Institut für Chemische Verfahrenstechnik der Univ. Stuttgart, Böblinger Str. 72,7000 Stuttgart 1 .
}

wird das vollständige Herauswandern dadurch verhindert, daß die Strömungsrichtung mit einer geeigneten Frequenz umgeschaltet wird. Innerhalb einer Periode wandert die Reaktionszone ein Stück in Strömungsrichtung; das kalte zuströmende Gas kühlt dabei den Eingangsbereich des Katalysators ab. Nach Umschalten der Strömungsrichtung wird der gerade abgekühlte Bereich durch die entgegengesetzt wandernde Temperaturfront wieder aufgeheizt: Eintritts- und Austrittsbereich des Katalysators wirken also als RegeneratorWärmeaustauscher.
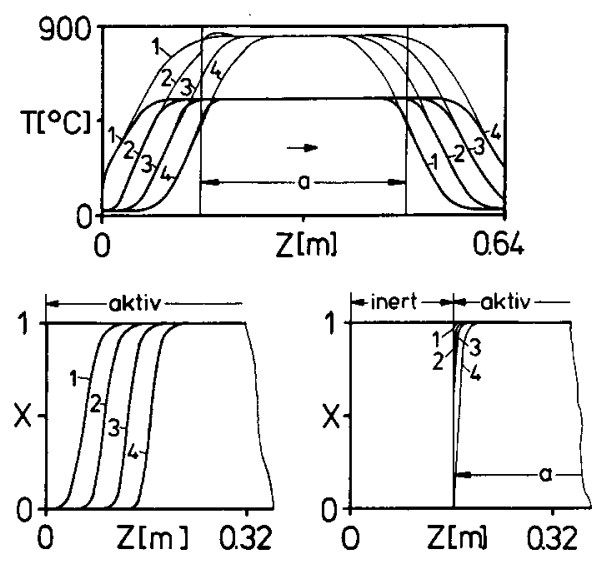

Abb. 2. Temperatur- und Umsatzprofile während einer Schaltperiode. Dicke Kurven: ohne inerte Randzonen, dünne Kurven: mit inerten Randzonen und Katalysator in a. Profile nach $1 \mathrm{~s}(1), 20 \mathrm{~s}(2)$, $40 \mathrm{~s} \mathrm{(3)}$ und $60 \mathrm{~s} \mathrm{(4)} \mathrm{nach} \mathrm{Umschalten.}$

\begin{abstract}
Ausgehend von einem Startprofil wird der eingeschwungene Zustand erst nach einer großen Zahl yon Umschaltungen erreicht. Im eingeschwungenen Zustand wandern die Temperatur- und Umsatzprofile in einer Periode zwischen den in Abb. 1a gezeigten Positionen hin und her. Die Reaktorablauftemperatur zeigt einen sägezahnförmigen Verlauf (Abb. 1b). Auf diese Weise wird die im Reaktor freigesetzte Wärme abgeführt.

Nur dieser Betrieb im eingeschwungenen Zustand sei im folgenden betrachtet. Die gezeigten Temperatur- und Umsatzprofile entsprechen jeweils dem Zustand kurz vor Umschalten der Strömungsrichtung, die durch einen Pfeil markiert ist.

Wie in [7] im einzelnen beschrieben, wirken sich die Betriebsparameter von Reaktor und Reaktion in einer zum Teil unerwarteten Weise auf Form und Maximaltemperatur des stationären Betriebs aus. Ursache ist die direkte Kopplung von Regenerator-Wärmeaustausch, chemischer Reaktion und Wärmespeicherung im Katalysator. Dabei ist die Maximaltemperatur im Katalysator um so höher, je weiter innen die Reaktionswärme freigesetzt wird, d. h. je weniger Reaktion in den beiden Endstücken des Katalysators stattfindet. So führen sinkender Stoßfaktor der Reaktion, sinkender Druck (bei gleichem Massenanteil), niedrigere Aktivierungsenergie oder inerte Randzonen wachsender Länge zunächst zu steigender Maximaltemperatur. Charakteristisch ist, daß die Steigung der Temperaturprofile in diesen Fällen nahezu gleich bleibt, obwohl sich die Umsatzprofile drastisch unterscheiden. Das zeigt exemplarisch Abb. 2 am Vergleich mit und ohne inerte Randzonen.
\end{abstract}

\section{Temperaturführungskonzepte}

Für den praktischen Betrieb des Oxidationsreaktors mit periodischem Wechsel der Strömungsrichtung sind geeignete Temperaturführungskonzepte erforderlich, die verhindern, daß bei zu niedriger 
Gasbeladung die Reaktion verlöscht und bei zu hoher Gasbeladung Grenztemperaturen überschritten werden. Das Verlöschen läßt sich nur über eine zusätzliche Wärmezufuhr - am einfachsten über eine geregelte Stützgas-Zumischung - erreichen.

Zur Vermeidung von Übertemperaturen sind eine Reihe von Maßnahmen möglich. Die wirkungsvollsten bestehen in einer Wärmeabfuhr direkt aus dem Inneren des Reaktors. Naheliegend sind ein in der Mitte des Reaktors eingebauter Wärmeaustauscher oder die von $\mathrm{Ma}$ tros $\mathrm{u}$. a. vorgeschlagene Führung des Gasstromes über einen externen Wärmeaustauscher [2]. In beiden Fällen kann aus Brenngas mit sehr nicdrigem Heizwert (adiabate Temperatur-Erhöhung um $100{ }^{\circ} \mathrm{C}$ ) Wärme auf hohem Temperaturniveau ausgekoppelt werden. Will man den apparativen Aufwand des Wärmeaustauschs vermeiden, so besteht eine einfache Möglichkeit zur Begrenzung der Maximaltemperatur darin, über einen Seitenabzug einen Teilstrom des ausreagierten Gases aus der Reaktormitte abzuziehen. Abb. 3 zeigt, wie sich dic Maximaltemperatur im Reaktor durch die Höhe des Gasabzugs in weiten Grenzen beeinflussen läßt.

\section{Modellrechnungen und Versuchsergebnisse}

Die in den Abb. 1 bis 3 gezeigten Ergebnisse stammen aus Modellrechnungen mit einem quasihomogenen, örtlich eindimensionalen Dispersionsmodell. Die Daten beziehen sich auf einen keramischen Monolith-Katalysator, wie er zur Autoabgas-Oxidation eingesetzt wird.

In einer Versuchsanlage im Labormaßstab (Reaktorlänge einschließlich inerter Randzonen $0,84 \mathrm{~m}$, Reaktordurchmesser $5 \mathrm{~cm}$ ) wurden edelmetallbeschichtete Autoabgas-Katalysatoren und ein mit CuMn beschichteter Monolith mit und ohne inerte Randzonen sowie mit und ohne Seitenabzug untersucht. Als Modellsubstanzen für Luftschadst offe dienten Methan, Propan und Propen. Die Ergebnisse entsprechen in ihrem qualitativen Verhalten denen der Simulationsrechnungen.

Die mit Edelmetall-Katalysatoren erreichten Querschnittsbelastungen liegen bei $2 \mathrm{~kg} \mathrm{Luft} / \mathrm{m}^{2} \mathrm{~s}$ und entsprechen einer Reinigungsleistung von mehr als $7000 \mathrm{~m}^{3} \mathrm{Abluft} / \mathrm{h}$ pro $\mathrm{m}^{3}$ Reaktorvolumen (aktiver und inerter Monolith).

Der Firma Degussa AG sei für die Bereitstellung von MonolithOxidationskatalysatoren gedankt. Herrn Prof. Riekert und Herrn Dr.
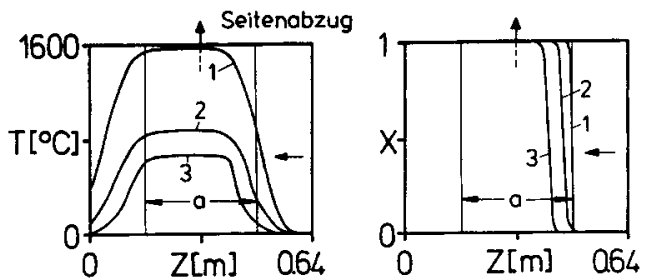

Abb. 3. Einfluß des Seitenabzugs auf die Temperaturprofile im eingeschwungenen Zustand $\left(\Delta T_{\text {ad }}=100^{\circ} \mathrm{C}\right) ; 1$ ohne Seitenabzug, 2 Gasabzug $10 \%, 3$ Gasabzug $15 \%$.

Kotter, Institut für Chemische Verfahrenstechnik der Univ. Karlsruhe, danken wir für die Beschichtung eines Monolith-Trägers mit ihrem CuMn-Katalysator.

Eingegangen am 28. Juni 1988

\section{Literatur}

[1] Boreskov, G. K.; Matros, Y. S.: Catal. Rev. Sci. Eng. 25 (1983) S. $551 / 590$.

[2] Matros, Y. S., u. a.: Vortrag auf dem Achema-Kongreß, Frankfurt/M. 1988

[3] Wojciechowski, J.: P 0037119 (1981).

[4] Wicke, E.; Vortmeyer, D.: Ber. Bunsenges. Phys. Chem. 63 (1959) S. $145 / 152$.

[5] Gilles, E. D.: Chem.-Ing.-Tech. 49 (1977) S. 142/149.

[6] Eigenberger, G.: Chem.-Ing.-Tech. 50 (1978) S. 924/933.

[7] Eigenberger, G.; Nieken, U.: 10th Int. Symp. on Chem. Reaction Eng. (ISCRE 10), Basel 1988, to be published in Chem. Eng. Sci.

Schlüsselworte: Abluft, Oxidation, Katalysatoren, Monolithe, Luftreinhaltung.

Das vollständige Manuskript dieser Arbeit umfaBt 25 Seiten mit 18 Abbildungen und 14 Literaturzitaten. Es ist als Fotokopie oder Mikrofiche MS 1715/88 erhältlich. Bestellkarten finden Sie am Schluß dieses Heftes. 